\title{
Facteurs socioéconomiques et de mode de vie associés à l'obésité chez les enfants d'âge scolaire fréquentant les écoles primaires privées de Cotonou
}

\author{
Joelle A. FLENON, Comlan Evariste S. MITCHIKPE* et \\ Djidjoho Joseph HOUNHOUIGAN
}

\author{
Ecole de Nutrition et des Sciences et Technologies Alimentaires, \\ Faculté des Sciences Agronomiques, Université d'Abomey-Calavi, Abomey-Calavi, Bénin. \\ *Auteur correspondant ; E-mail: evaristemitchikpe@yahoo.fr; Tel: +22997145214 / 64098434
}

\section{RESUME}

L'obésité constitue un facteur de risque de maladies non transmissibles et de mortalité. Cette étude a pour objectif d'évaluer la prévalence de l'obésité et les facteurs socioéconomiques et de mode de vie qui y sont associés. Des données anthropométriques, de consommation alimentaire, d'activité physique et de statut socioéconomique des ménages ont été collectées sur 100 enfants de 7 à 13 ans fréquentant des écoles primaires privées dans la ville de Cotonou. La prévalence de l'obésité est de $16 \%$ et celle de la maigreur $24 \%$. Les caractéristiques anthropométriques et les apports nutritionnels des enfants obèses sont statistiquement supérieurs à ceux des enfants non-obèses. La consommation des fruits et légumes est faible chez tous les enfants. Toutefois, il y a une meilleure diversification alimentaire ainsi qu'une prépondérance de produits sucrés et animaux chez les enfants obèses. Ces derniers sont également caractérisés par une réduction des pratiques d'activités physiques et plus de temps consacrés aux activités sédentaires. L'obésité chez les enfants est liée au niveau socioéconomique des ménages. La sensibilisation sur la malnutrition, la formation sur l'alimentation et un bon système de surveillance pourraient prévenir les effets néfastes de la malnutrition chez les enfants d'âge scolaire au Bénin.

(C) 2018 International Formulae Group. All rights reserved.

Mots clés: Obésité, mode de vie, état nutritionnel, consommation alimentaire, activités physiques, enfants d'âge scolaire.

\section{Socioeconomic and lifestyle factors associated with obesity among school-age children attending private primary schools in Cotonou}

\begin{abstract}
Obesity is known to be a risk factor of non-communicable diseases and mortality. The objective of this study was to estimate the prevalence of obesity and associated socioeconomic and lifestyle factors. Data on anthropometry, food consumption, physical activity and socioeconomic status of the households were collected on 100 children of 7 to 13 years old, attending private primary schools in the city of Cotonou. Prevalence of obesity is $16 \%$ and that of thinness is $24 \%$. Anthropometric characteristics and nutritional intakes of obese children are statistically higher than that of non-obese children. Fruits and vegetables consumption are low for all children. However, there is a better food diversification as well as an increased intake of sweet and animal
\end{abstract}


products among obese children. These children are also characterized by a reduced practice of physical activities and more time dedicated to sedentary activities. There is a relation between obesity among the children and socioeconomic level of the households. Sensitization on malnutrition, training on good feeding practices and a good monitoring system could prevent the negative impacts of malnutrition on school-age children in Benin.

(C) 2018 International Formulae Group. All rights reserved.

Keywords: Obesity, lifestyle, nutritional status, food consumption, physical activities, school-age children.

\section{INTRODUCTION}

Les changements des habitudes alimentaires et des modes de vie liés à l'urbanisation sont les principales causes du surpoids et de l'obésité au sein des populations urbaines. L'obésité constitue un facteur de risque de maladies non transmissibles telles que le diabète et les maladies cardio-vasculaires à un âge précoce. Elle produit chez les enfants atteints, des effets indésirables tels que des difficultés respiratoires, un risque accru de fracture, de l'hypertension, des marqueurs précoces des maladies cardiovasculaires, une résistance à l'insuline, et des effets psychologiques (OMS, 2010 ; Afrifa-Anane et al., 2015).

Le surpoids et l'obésité sont en train de prendre de l'ampleur dans les pays en développement qui, dans le même temps, font face à une prévalence élevée de la malnutrition par carence (OMS, 2014; Ntandou, 2009). Ce double fardeau nutritionnel fragilise davantage le système de santé surtout en termes d'insuffisance de moyens matériel et économique pour faire face à la prise en charge de l'obésité et de ses complications (OMS, 2003a ; Ministère de la Santé du Bénin, 2008).

Au Bénin, la surcharge pondérale a été documentée chez les femmes en âge de procréer, les adultes et les personnes âgées. La prévalence de la surcharge pondérale est estimée à $17 \%$ et celle de l'obésité à $10 \%$ chez les femmes de 18 à 69 ans (Ministère de la Santé du Bénin, 2016). A Cotonou en 2008, la prévalence de l'obésité était estimée à 18,1\% parmi les femmes de 15 à 49 ans et à $28 \%$ parmi celles âgées de 25 à 60 ans $(\mathrm{GdB}$, 2009 ; Sodjinou et al., 2008). En revanche, les données sur la prévalence du surpoids et de l'obésité chez les enfants en milieu scolaire sont quasi inexistantes.

La présente étude a pour objectif d'évaluer la prévalence de l'obésité et d'étudier les facteurs socioéconomiques et de mode de vie associés à ce type de malnutrition chez les enfants d'âge scolaire fréquentant les écoles primaires privées dans la ville de Cotonou.

\section{MATERIEL ET METHODES Cadre d'étude}

L'étude a été réalisée dans la ville de Cotonou, capitale économique du Bénin. Comme la plupart des villes des pays en développement, Cotonou a connu une urbanisation rapide ces dernières décennies. Celle-ci a pu induire un changement significatif dans les habitudes alimentaires et le mode de vie de ses habitants (Sodjinou et al., 2008 ; Ntandou, 2009). Dans cette ville, l'enseignement primaire est délivré par des écoles publiques mais aussi par les écoles privées offrant des services de différents standings.

\section{Plan d'étude}

Il s'agit d'une étude transversale descriptive et analytique mise en œuvre au cours de l'année académique 2010-2011 sur un échantillon d'enfants de 7 à 13 ans fréquentant les Cours Moyens $1^{\text {ère }}$ et $2^{\text {ème }}$ années (CM1 et CM2) de 5 écoles primaires privées de Cotonou. L'état nutritionnel des enfants a été apprécié grâce à l'anthropométrie. Les apports nutritionnels ont été évalués grâce à une enquête de consommation alimentaire. Les activités 
physiques des enfants ainsi que les statuts socioéconomiques des ménages ont été évalués à l'aide de questionnaires.

\section{Echantillonnage}

L'échantillonnage a été réalisé en utilisant la méthode d'échantillonnage aléatoire stratifiée. Ainsi, les écoles privées régulièrement inscrites au Ministère des Enseignements Maternel et Primaire pour l'année scolaire 2009-2010 ont été répartie en cinq catégories sur la base des frais de scolarité. Il s'agit de : Catégorie1 $(\mathrm{C} 1)=$ frais de scolarité $\leq 48000$ FCFA ; C2 frais de scolarité compris entre 48000 et 55000 FCFA ; $\mathrm{C} 3$ = frais de scolarité compris entre 55000 et 85000 FCFA ; $\mathrm{C} 4=$ frais de scolarité compris entre 85000 et 165000 FCFA; C5 = frais de scolarité $>165000$ FCFA. Cinq écoles ont été sélectionnées de façon aléatoire dans chaque catégorie et à l'intérieur de chacune des strates, une école a été choisie également aléatoirement pour faire partie de cette étude. Dans chacune de ces écoles, les classes de CM1 et de CM2 ont été retenues pour participer à l'étude.

La prévalence de l'obésité au sein du groupe cible calculée lors d'une étude pilote réalisée plus tôt a permis d'estimer la taille de l'échantillon à 100 enfants. Ainsi, 20 enfants ont été sélectionnées de façon aléatoire par école à partir de la liste de ceux dont les parents sont favorables à leur participation.

\section{Etat nutritionnel des enfants}

Les mesures anthropométriques ont été prises sur les enfants dans la matinée, au sein de leurs écoles respectives. L'état nutritionnel des enfants a été évalué grâce aux variables anthropométriques ci-après :

Poids : Le poids a été déterminé à l'aide d'un pèse-personne électronique Uniscale de type SECA, de portée $150 \mathrm{~kg}$, à $0,1 \mathrm{~kg}$ près.

Taille : La taille a été mesurée à l'aide d'une toise Shorr de précision $0,1 \mathrm{~cm}$.

Epaisseurs des plis cutanés : Les plis cutanés tricipital et bicipital ont été mesurés à l'aide d'un compas de type 'Harpenden'. La mesure a été réalisée sur le bras gauche avec une précision de $0,2 \mathrm{~mm}$.

Tour du bras : Le tour du bras a été mesuré sur le bras gauche à l'aide d'un mètre ruban. La lecture a été faite à $0,1 \mathrm{~cm}$ près.

Périmètre abdominal ou tour de taille: Les enfants ont été mesurés les pieds écartés d'environ $25 \mathrm{~cm}$, au niveau du point situé à égale distance du bord inférieur de la dernière côte et de la crête iliacale. La mesure a été effectuée à l'aide d'un mètre ruban à $0,1 \mathrm{~cm}$ près.

Tour de hanches: Le tour de hanche a été mesuré sur les enfants en position debout, les bras le long du corps et les pieds joints. La mesure a été prise à l'aide d'un ruban d'insertion, avec une précision de $0,1 \mathrm{~cm}$.

Indice de Masse Corporelle : Le poids et la taille des enfants ont servi à calculer l'Indice de Masse Corporelle (IMC). Les prévalences du surpoids, de l'obésité ainsi que de la maigreur (légère, modérée et sévère) ont été déterminées en utilisant les valeurs seuils proposés par Cole et al. (2000) et Cole et al. (2007).

\section{Evaluation du modèle alimentaire des enfants}

Les modèles alimentaires des enfants ont été caractérisés grâce à la proportion d'enfants ayant consommé les groupes d'aliments considérés, le nombre moyen de repas habituellement pris par les enfants, les proportions d'enfants ayant de l'attrait pour les friandises et ceux qui consomment habituellement leurs repas devant la télévision. Les données ont été collectées grâce à un questionnaire.

\section{Evaluation des apports nutritionnels}

Une enquête de consommation alimentaire a été réalisée pour évaluer les apports nutritionnels des enfants en utilisant la méthode de rappel des $24 \mathrm{~h}$ en un seul passage (Gibson et al., 2008). Elle a permis de décrire, pour chaque enfant, les aliments consommés à la maison et à la cantine scolaire, leurs origines et le moment de la consommation 
ainsi que les ingrédients contenus dans ces aliments. Les quantités consommées ont été estimées en unités de mesures ménagères. Ces dernières ont été ensuite converties en unités du système international. Les aliments achetés par les enfants ont été également identifiés et les ingrédients contenus dans ces derniers ont été déterminés par interview auprès des vendeuses.

Les apports en macro et en micronutriments des aliments consommés ont été calculés après avoir mis-à-jour la base de données de composition alimentaire du programme World Food Dietary Assesment System, version 2, de la FAO. Les teneurs en nutriments des plats préparés ont été calculées en appliquant les recettes établies au cours de l'enquête ainsi que les facteurs de dilution ou de concentration. Les contributions moyennes des glucides, protéines, lipides, graisses saturées et polyinsaturées à l'apport énergétique total ont été également déterminées.

\section{Qualité des apports alimentaires}

La qualité des apports alimentaires a été appréciée par le score moyen de diversité alimentaire et le niveau de diversité alimentaire (FAO, 2011 ; FAO, 2013).

Le niveau de diversité alimentaire a été déterminé grâce à l'utilisation des quartiles. Ainsi, le niveau de diversité alimentaire est considéré comme étant faible, moyen, élevé ou très élevé, respectivement pour, 5 à 6 groupes d'aliments ; 7 groupes d'aliments ; 8 groupes d'aliments; et 9 à 12 groupes d'aliments.

\section{Activités physiques}

Les activités physiques des enfants ont été évaluées grâce à un questionnaire qui a renseigné la pratique et la durée quotidienne ou hebdomadaire de certaines activités. Il s'agit de: se rendre à l'école, participation aux activités récréatives, au cours d'éducation physique et sportive (EPS), aux activités physiques et sportives structurées effectuées en dehors de l'école, regarder la télévision, jouer au jeux vidéo et à l'ordinateur, et enfin, le sommeil. Les temps passés devant la télévision, les jeux vidéo ou l'ordinateur et pour le temps de sommeil, durant les jours ouvrables ou en week-end et jours fériés ont été évalués.

\section{Statut socioéconomique des ménages des enfants}

Le statut socioéconomique des ménages a été déterminé grâce à la profession du père, au montant journalier de l'argent de poche de l'enfant, et aux informations sur la possession de biens par le ménage. Un ensemble de questions relatives à la possession de biens a servi à l'estimation du niveau de revenu (Houweling et al., 2003 ; Sodjinou et al., 2008).

Un score de possession de biens a été calculé en faisant la somme arithmétique des scores individuels obtenus pour chacune des dix (10) variables qui constituent les biens possédés par le ménage. Les scores de possession ont été regroupés en terciles de la manière suivante $: \leq 3,5$ niveau de possession faible ; 3,5 - 6,5 niveau de possession moyen et $\geq 6,5$ niveau élevé de possession de biens.

La profession des parents a été aussi répartie en cinq catégories à savoir : cadre, employé, commerçants et chefs d'entreprise, artisans et professions intermédiaires.

\section{Ethique}

Des autorisations de recherche ont été obtenues auprès de Direction Départementale de l'Enseignement Maternel et Primaire de l'Atlantique-Littoral et des directeurs des écoles avant la mise en œuvre de cette étude. Des consentements pour la participation des enfants ont été également signés par les parents. Les mesures anthropométriques prises ainsi que les informations collectées ont été expliquées aux enfants et ont été traitées de façon confidentielle.

\section{Analyse statistique}

Les variables mesurées ont été décrites

à l'aide des statistiques descriptives. Les 
valeurs hors normes ont été vérifiées puis exclues de l'analyse si nécessaire. Les moyennes entre les groupes d'enfants ont été comparées en utilisant le test $t$ de student ou le test de Mann Withney et les proportions ont été comparées à l'aide du test d'indépendance $\mathrm{Chi}^{2}$. Les analyses ont été effectuées avec le logiciel SPSS, version16. Les tests sont reconnus comme étant statistiquement significatifs pour les valeurs de $p<0,05$.

\section{RESULTATS}

\section{Caractéristiques des sujets}

L'âge moyen des enfants de l'étude est de 9,3 $\pm 0,1$ ans et aucune différence significative n'est observée entre enfants obèses et enfants non-obèses, ou entre garçons et filles (Tableau 1).

Le Tableau 1 montre que les valeurs moyennes de la taille $(141,4 \pm 1,7 \mathrm{~cm})$, du poids $(45,6 \pm 2,5 \mathrm{~kg})$, du tour de bras $(26,0 \pm$ $0,8 \mathrm{~cm})$, du tour de taille $(75,6 \pm 2,2 \mathrm{~cm})$, du tour de hanche $(87,8 \pm 2,2 \mathrm{~cm})$, du pli cutané tricipital $(22,2 \pm 1,8 \mathrm{~mm})$, du plis cutané bicipital $(17,7 \pm 2,1 \mathrm{~mm})$ et de l'IMC $(22,9 \pm$ $\left.0,8 \mathrm{~kg} / \mathrm{m}^{2}\right)$ des enfants obèses sont significativement supérieures à celles des enfants non-obèses $(137,3 \pm 0,8 \mathrm{~cm} ; 29,6 \pm$ $0,6 \mathrm{~kg} ; 18,8 \pm 0,2 \mathrm{~cm} ; 58,7 \pm 0,5 \mathrm{~cm} ; 70,0 \pm$ $0,7 \mathrm{~cm} ; 8,8 \pm 0,4 \mathrm{~mm} ; 6,0 \pm 0,3 \mathrm{~mm}$ et 15,5 $\left.\pm 0,2 \mathrm{~kg} / \mathrm{m}^{2}\right)(\mathrm{p}<0,05)$.

Aucune différence significative n'a été observée entre garçons et filles pour ce qui concerne les variables et indices anthropométrique $(\mathrm{p}>0,05)$.

\section{Prévalence de la malnutrition}

La prévalence de la maigreur au sein des enfants est de $24 \%$ et se manifeste surtout sous forme de maigreur légère (Tableau 2). La prévalence de l'obésité est de $16 \%$. La prévalence de la maigreur est plus élevée chez les filles $(31 \%)$ que chez les garçons $(17 \%)$, tandis que la prévalence de l'obésité est plus élevée chez les garçons (23\%) que chez les filles $(10 \%)$. Cependant, ces différences ne sont pas statistiquement significatives.

\section{Caractéristiques du modèle alimentaire des enfants}

Le profil de consommation alimentaire des enfants montre que les groupes d'aliments: huiles et graisses; légumes ; céréales ; épices, condiments et boissons, ont été consommés par la quasi-totalité des enfants (99\% à 100\%) (Figure 1). Les viandes et produits carnés, ont été consommés par $61 \%$ des enfants. Le sucre et les produits sucrés ont été consommés par $51 \%$, tandis que les fruits ont été consommés par seulement $20 \%$ des enfants. Seules les proportions d'enfants ayant consommé les viandes et produits carnés sont statistiquement différentes entre les deux groupes, avec une consommation des enfants obèses supérieure à celle des enfants non-obèses $(p<0,05)$.

La fréquence moyenne journalière de repas consommés par l'ensemble des enfants est de 4,4 $\pm 0,1$ (Tableau 3). Cette fréquence est statistiquement plus élevée chez les enfants obèses $(5,4 \pm 0,3)$ que chez les enfants non-obèses $(4,7 \pm 0,1) \quad(\mathrm{p}<0,05)$. Aucune différence significative n'a été observée entre les enfants obèses et non-obèses pour ce qui concerne les proportions d'enfants qui mangent les repas du midi à la cantine scolaire, ceux qui mangent la friandise ou ceux qui consomment leurs repas devant la télévision.

\section{Apports moyens en macro- et micronutriments}

L'apport moyen en énergie est de $7,8 \pm 6,9$ MJ pour l'ensemble des enfants (Tableau 4). Les apports moyens en glucides, lipides et protéines sont estimés respectivement à $181,4 \pm 125,4 \mathrm{~g} ; 104,4 \pm 120,6$ $\mathrm{g}$ et $49,1 \pm 38,4 \mathrm{~g}$. Les apports de graisses saturées et polyinsaturées, de cholestérol et de fibres sont évalués respectivement à, $21,7 \pm 24,3 \mathrm{~g} ; 32,1 \pm 37,8 \mathrm{~g} ; 71,3 \pm 73 \mathrm{~g}$ et $32,1 \pm 34,6$ g. Les apports en macro et micronutriments des enfants obèses sont statistiquement supérieurs à ceux des enfants non-obèses $(p<0,05$ et $p<0,01)$. 


\section{Contribution des glucides, protéines et lipides à l'apport énergétique total}

Dans l'ensemble, les glucides totaux, les protéines et les lipides contribuent pour, $47 \%, 13 \%$ et $40 \%$, respectivement des apports énergétiques totaux (Figure 2). La contribution des lipides à l'apport énergétique total est plus élevée chez les enfants obèses (51\%) que chez les enfants non-obèses (38\%), avec une différence statistiquement significative $(p<0,05)$. En revanche, pour les protéines et les glucides, leur contribution à l'apport énergétique total est plus élevée chez les enfants non-obèses $(13 \%$ et $49 \%$, respectivement) que chez les enfants obèses $(10 \%$ et $39 \%$, respectivement) $(\mathrm{p}<0,05)$.

Les graisses saturées et polyinsaturées représentent environ 9 et $12 \%$ de l'apport énergétique total (Figure 3). Chez les enfants obèses, elles représentent environ 11 et $15 \%$, respectivement, alors que chez les enfants non-obèses, elles représentent environ 8 et $11 \%$, respectivement. Il existe une différence significative entre la contribution des graisses saturées à l'apport énergétique total chez les enfants obèses et non-obèses $(p<0,05)$. Il en est de même pour la contribution des graisses polyinsaturées $(\mathrm{p}<0,01)$

\section{Score moyen et niveau de diversité alimentaire des enfants}

Le score moyen de diversité alimentaire est 7,7 $\pm 0,1$. Ce score est 8,25 \pm 0,3 et 7,6 $\pm 0,1$ pour les enfants obèses et nonobèses, respectivement. Bien que l'apport alimentaire des enfants obèses soit plus diversifié que celui des enfants non-obèses, il n'existe pas une différence significative entre les groupes d'enfants $(p=0,05)$.

Le niveau de diversité alimentaire (Figure 4) montre que 14\% des enfants nonobèses ont une faible diversité alimentaire. La proportion d'enfants obèses ayant une diversité alimentaire très élevée $(43,8 \%)$ est le double de celle des enfants non-obèses $(21,4 \%)$.
Description et durées moyennes des principales activités physiques exercées par les enfants

Plus de la moitié (54\%) des enfants viennent à l'école en moyens motorisés (Tableau 5). Seulement 6\% des enfants obèses viennent à l'école à pieds contre $54 \%$ des enfants non-obèses, la différence étant statistiquement significative $(p<0,05) . \quad \mathrm{La}$ durée moyenne du trajet effectuée par les enfants venant à pieds est d'environ trois quart d'heure pour une journée d'école.

Seulement $40 \%$ des enfants participent aux activités physiques structurées (activités sportives) hors école, la proportion étant plus élevée chez les enfants obèses $(62,5 \%)$ que chez les enfants non-obèses $(35,7 \%)$ avec une différence statistiquement significative $(\mathrm{p}<0,05)$. Il n'y pas de différence statistiquement significative entre la durée moyenne des activités physiques structurées hors école des enfants obèses et des enfants non-obèses.

Parmi les deux groupes d'enfants, en moyenne $2 \mathrm{~h} 20$ à $2 \mathrm{~h} 30 \mathrm{~min}$ sont consacrées chaque jour de la semaine à la télévision. Les enfants obèses passent quotidiennement environ $1 \mathrm{~h}$ de temps devant les jeux vidéo ou l'ordinateur alors que les enfants non-obèses y passent environ $30 \mathrm{~min}$. La durée moyenne du sommeil des enfants est d'environ $9 \mathrm{~h}$ en jours ouvrables. Pendant ces jours, les enfants obèses ont tendance à dormir en moyenne 15 min de plus que les enfants non-obèses tandis qu'en week-end et jours fériés, les enfants obèses ont tendance à dormir en moyenne 20 min de plus que les enfants non-obèses. Toutefois, les différences ne sont pas statistiquement significatives.

\section{Caractéristiques socioéconomiques des ménages des enfants}

Les enfants reçoivent en moyenne $186,3 \pm 12,5$ FCFA par jour comme argent de poche (Tableau 6). Le montant moyen journalier reçu par les enfants obèses $(328,1 \pm$ 36,2 FCFA) est plus élevé que celui reçu par les enfants non-obèses $(159,2 \pm 11,0$ FCA $)$, la 
différence étant statistiquement significative $(\mathrm{p}<0,05)$.

Environ $69 \%$ des parents d'enfants obèses ont un niveau de possession de biens élevé, alors que seulement $29 \%$ des parents d'enfants non-obèses sont concernés, la différence étant statistiquement significative $(\mathrm{p}<0,05)$.

Chez les enfants obèses, environ $80 \%$ des parents sont des cadres, des commerçants ou chefs d'entreprise, alors que chez les enfants non-obèses, cette proportion est de 49\%. Il existe également une différence statistiquement significative entre les professions des pères d'enfants obèses et des pères d'enfants non-obèses $(\mathrm{p}<0,05)$.

Tableau 1 : Caractéristiques anthropométriques des sujets.

\begin{tabular}{|c|c|c|c|c|c|}
\hline & Ensemble & $\begin{array}{c}\text { Enfants } \\
\text { obèses }\end{array}$ & $\begin{array}{c}\text { Enfants } \\
\text { non-obèses }\end{array}$ & Garçons & Filles \\
\hline $\mathbf{N}$ & 100 & 16 & 84 & 48 & 52 \\
\hline Age (ans) & $9,3 \pm 0,1$ & $9,1 \pm 0,3$ & $9,3 \pm 0,1$ & $9,2 \pm 0,1$ & $9,3 \pm 0,1$ \\
\hline Taille $(\mathrm{cm})$ & $138,0 \pm 0,7$ & $\begin{array}{c}141,4 \pm \\
1,7 *\end{array}$ & $137,3 \pm 0,8$ & $138,5 \pm 5$ & $\begin{array}{c}137,5 \pm \\
1,0\end{array}$ \\
\hline Poids (kg) & $32,1 \pm 0,9$ & $45,6 \pm 2,5^{*}$ & $29,6 \pm 0,6$ & $33,7 \pm 1,4$ & $30,6 \pm 0,9$ \\
\hline Tour du bras (TB) (cm) & $20,0 \pm 0,3$ & $26,0 \pm 0,8^{*}$ & $18,8 \pm 0,2$ & $20,3 \pm 0,4$ & $19,6 \pm 0,4$ \\
\hline Tour de taille (TT) $(\mathrm{cm})$ & $61,4 \pm 0,8$ & $75,6 \pm 2,2^{*}$ & $58,7 \pm 0,5$ & $63,1 \pm 1,4$ & $59,8 \pm 0,9$ \\
\hline Tour de hanche $(\mathrm{TH})(\mathrm{cm})$ & $72,9 \pm 0,9$ & $87,8 \pm 2,2^{*}$ & $70,0 \pm 0,7$ & $74,4 \pm 1,4$ & $71,5 \pm 1,1$ \\
\hline $\begin{array}{l}\text { Pli cutané tricipital (PCT) } \\
(\mathbf{m m})\end{array}$ & $11,0 \pm 0,6$ & $22,2 \pm 1,8^{*}$ & $8,8 \pm 0,4$ & $11,4 \pm 1,1$ & $10,6 \pm 0,7$ \\
\hline $\begin{array}{l}\text { Pli cutané bicipital }(\mathbf{P C B}) \\
(\mathrm{mm})\end{array}$ & $7,9 \pm 0,6$ & $17,7 \pm 2,1 *$ & $6,0 \pm 0,3$ & $8,5 \pm 1,1$ & $7,3 \pm 0,6$ \\
\hline $\operatorname{IMC}\left(\mathrm{kg} / \mathrm{m}^{2}\right)$ & $16,7 \pm 0,3$ & $22,9 \pm 0,8^{*}$ & $15,5 \pm 0,2$ & $17,4 \pm 0,6$ & $16,0 \pm 0,3$ \\
\hline TT/TH & $0,8 \pm 0,0$ & $0,9 \pm 0,0$ & $0,8 \pm 0,0$ & $0,8 \pm 0,0$ & $0,8 \pm 0,0$ \\
\hline
\end{tabular}

Moyenne \pm Ecart-type

* Différence statistiquement significative entre enfants obèses et enfants non obèses $(p<0,05)$.

Tableau 2 : Prévalence des différents types et formes de malnutrition au sein des enfants.

\begin{tabular}{lccc}
\hline & Ensemble & Garçons & Filles \\
\hline $\mathbf{N}$ & $\mathbf{1 0 0}$ & $\mathbf{4 8}$ & $\mathbf{5 2}$ \\
\hline Type de malnutrition & & & \\
$\quad$ Maigreur (\%) & 24,0 & 16,7 & 30,8 \\
Obésité (\%) & 16,0 & 22,9 & 9,6 \\
Degré de malnutrition & & & \\
$\quad$ Maigreur sévère (\%) & 3,0 & 0,0 & 5,8 \\
Maigreur modérée (\%) & 4,0 & 2,1 & 5,8 \\
Maigreur légère (\%) & 17,0 & 14,6 & 19,2 \\
Obésité degré 1 (\%) & 9,0 & 10,4 & 7,7 \\
Obésité degré 2(\%) & 7,0 & 12,5 & 1,9 \\
\hline
\end{tabular}




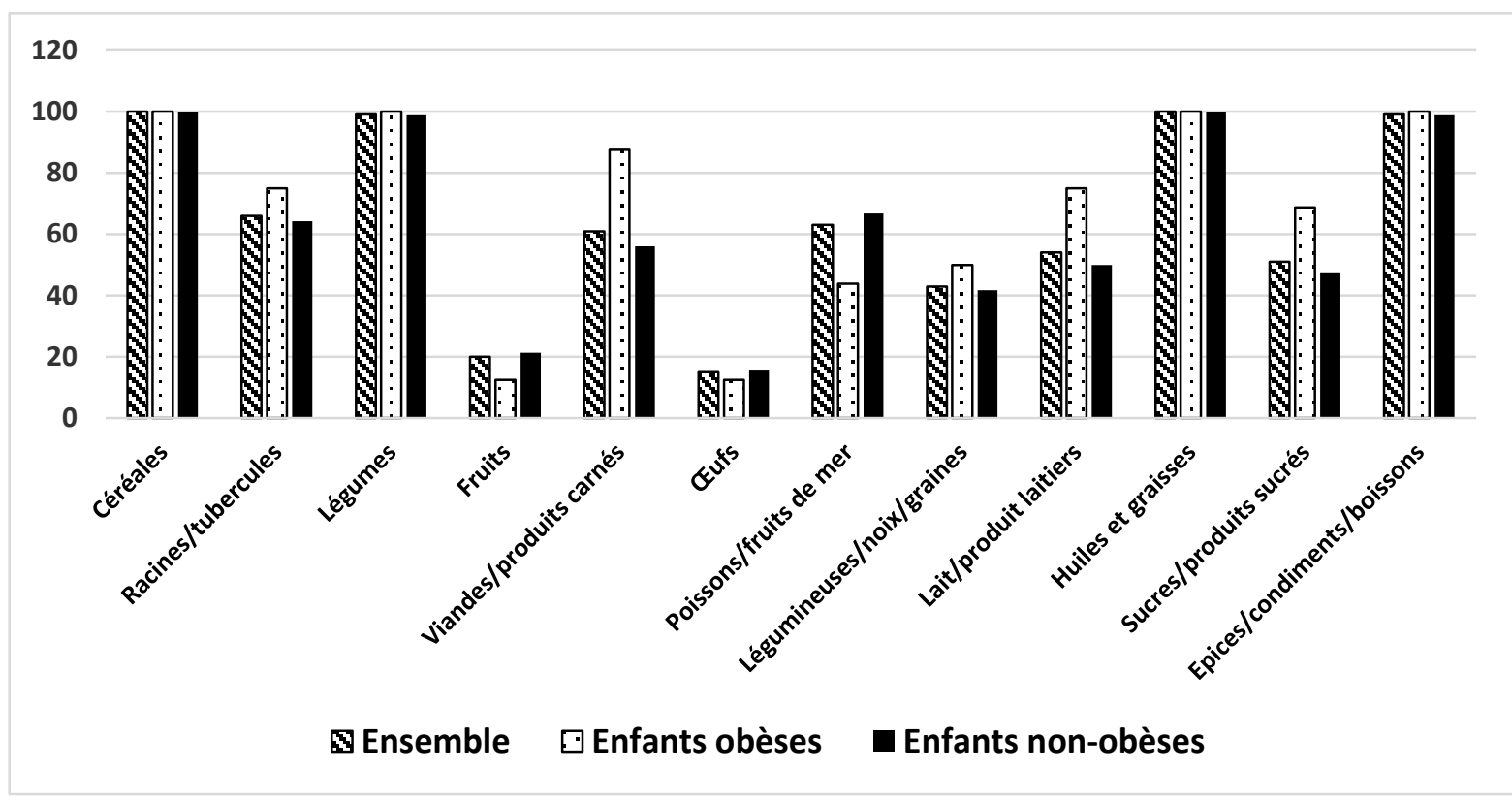

Figure 1: Proportion d'enfants de chaque groupe ayant consommé les différents groupes alimentaires $(\%)$.

Tableau 3: Fréquence moyenne journalière de repas et répartition des enfants selon leur comportement alimentaire.

\begin{tabular}{lccc}
\hline & Ensemble & Enfants Obèses & Enfants Non-obèses \\
\hline $\mathrm{N}$ & 100 & 16 & 84 \\
\hline Fréquence moyenne journalière de repas & $4,4 \pm 0,1$ & $5,4 \pm 0,3^{*}$ & $4,7 \pm 0,1$ \\
Fréquentation cantine scolaire (\%) & 38,0 & 50,0 & 35,7 \\
Friandise (\%) & 90,0 & 100,0 & 88,0 \\
Repas devant télévision $(\%)$ & 64,0 & 68,8 & 63,1 \\
\hline * significationent & &
\end{tabular}

* significativement plus élevée que chez les enfants non-obèses $(\mathrm{p}<0,05)$.

Tableau 4 : Apports alimentaires moyens en macro et micronutriments (moyenne \pm ET) des enfants.

\begin{tabular}{lccc}
\hline & Ensemble & Enfants Obèses & Enfants Non-obèses \\
\hline $\mathrm{N}$ & 94 & 16 & 78 \\
\hline Macronutriments & & & \\
Energie totale (MJ) & $7,8 \pm 6,9$ & $14,1 \pm 9,1 * *$ & $6,5 \pm 5,5$ \\
Glucides totaux (g) & $181,4 \pm 125,4$ & $273,0 \pm 165,0^{* *}$ & $162,6 \pm 107,7$ \\
Protéines (g) & $49,1 \pm 38,4$ & $73,0 \pm 42,6^{* *}$ & $44,2 \pm 35,9$ \\
Lipides totaux (g) & $104,4 \pm 120,6$ & $221,1 \pm 164,0^{* *}$ & $80,4 \pm 94,4$ \\
Graisses saturées (g) & $21,7 \pm 24,3$ & $45,0 \pm 31,5 * *$ & $16,9 \pm 19,6$ \\
Graisses polyinsaturées (g) & $32,1 \pm 37,8$ & $67,6 \pm 52,4 * *$ & $24,8 \pm 29,5$ \\
Cholestérol (mg) & $71,3 \pm 73,0$ & $108,6 \pm 71,0^{*}$ & $63,6 \pm 71,5$ \\
Fibres (g) & $32,1 \pm 34,6$ & $53,8 \pm 46,4^{*}$ & $27,7 \pm 30,1$
\end{tabular}


Micronutriments

Calcium (mg)

Fer $(\mathrm{mg})$

Zinc (mg)

Vitamine C (mg)

$\begin{array}{cc}335,1 \pm 380,7 & 558,6 \pm 481,7 * \\ 16,3 \pm 19,5 & 27,9 \pm 26,5^{*} \\ 6,4 \pm 5,6 & 10,8 \pm 6,6^{* *} \\ 111,7 \pm 144,8 & 213,7 \pm 201,1^{*}\end{array}$

$335,1 \pm 380,7$
$289,3 \pm 342,5$

$13,9 \pm 17,0$

$5,5 \pm 5,0$

$90,8 \pm 121,9$

ET= écart-type associé à la moyenne ;

* Différence statistiquement significativement entre enfants obèses et non-obèses $(p<0,05)$;

** Différence statistiquement significativement entre enfants obèses et non-obèses $(p<0,01)$.

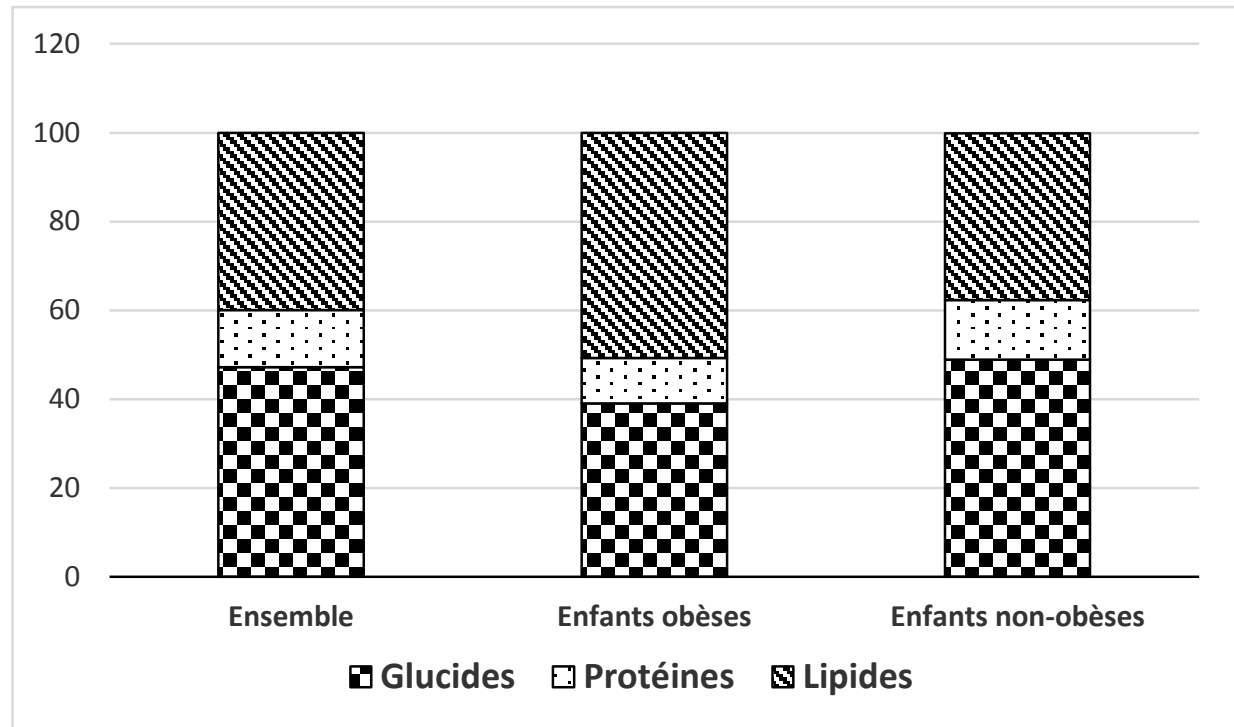

Figure 2 : Contribution (\%) des glucides, protéines et lipides à l'apport énergétique total.

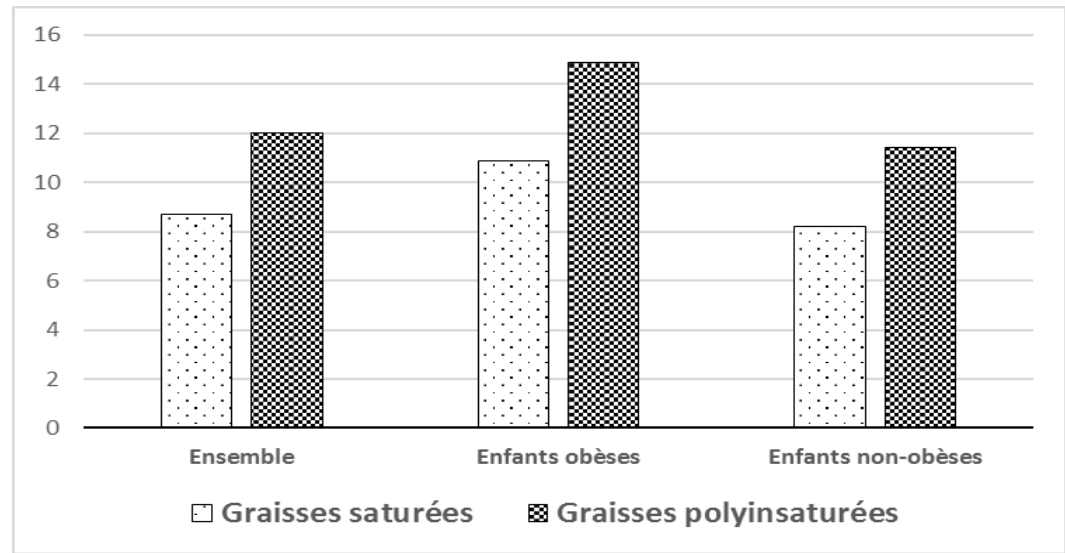

Figure 3 : Contribution (\%) des graisses saturées et polyinsaturées à l'apport énergétique total. 


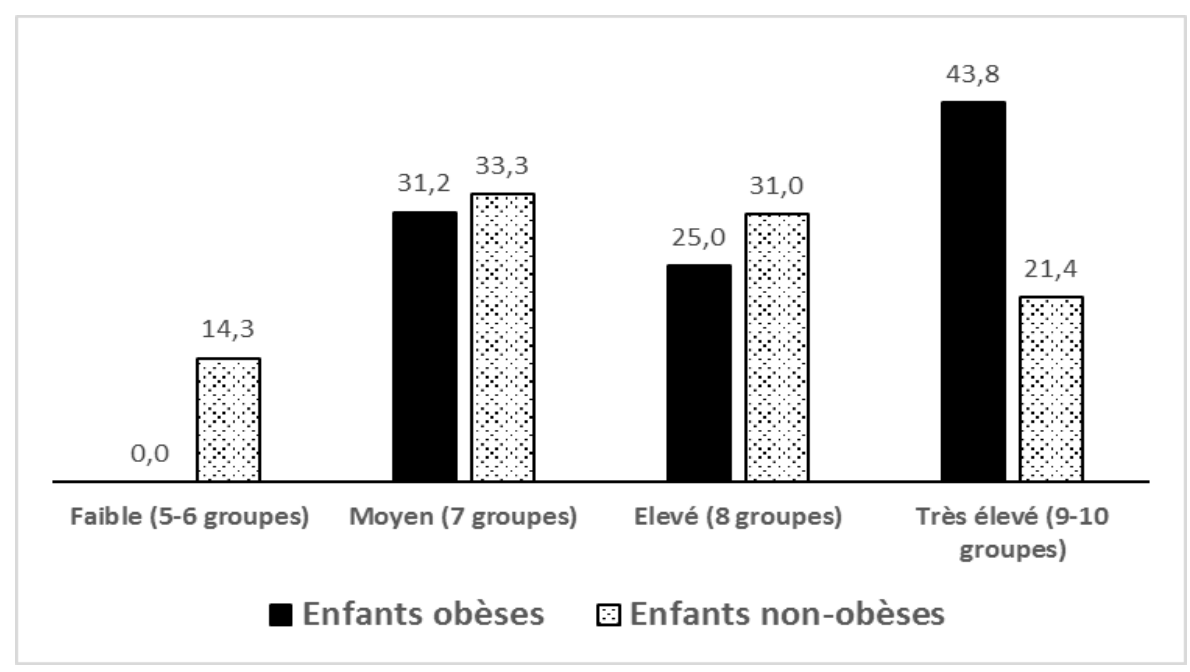

Figure 4: Répartition des proportions d'enfants de chaque groupe selon les niveaux de diversité alimentaire $(\%)$.

Tableau 5 : Pratiques des activités physiques par les enfants et durées moyennes.

\begin{tabular}{lccc}
\hline & Ensemble & $\begin{array}{c}\text { Enfants } \\
\text { Obèses }\end{array}$ & $\begin{array}{c}\text { Enfants } \\
\text { Non obèses }\end{array}$ \\
\hline $\mathrm{N}$ & 100 & 16 & 84 \\
\hline Pratiques des activités physiques intenses (\%) & & & \\
Marche à pieds pour aller à l'école & 46,0 & $6,2^{*}$ & 53,6 \\
Moyens motorisés pour aller à l'école & 54,0 & $93,8^{*}$ & 46,4 \\
Activités récréatives au sein de l'école & 93,0 & 93,8 & 92,9 \\
AP Structurées au sein de l'école & 63,0 & 75,0 & 60,7 \\
AP Structurées hors école & 40,0 & $62,5^{*}$ & 35,7 \\
Durées moyennes des activités physiques intenses & & & \\
Pour aller à l'école (min/jour) & $46,8 \pm 3,7$ & $20,0 \pm 0,0$ & $47,4 \pm 3,7$ \\
AP structurées hors école (min/semaine) & $128,6 \pm 13,2$ & $153,0 \pm 28,4$ & $120,5 \pm 14,8$ \\
Durées moyennes des activités sédentaires & & & \\
Télévision (min/jour) & $146,9 \pm 7,5$ & $140,6 \pm 13,1$ & $148,1 \pm 8,6$ \\
Ordinateur ou jeux vidéo (min/jour) & $36,2 \pm 5,5$ & $58,1 \pm 14,8$ & $31,9 \pm 5,9$ \\
Télévision + ordinateur en jours ouvrables (min/jour) & $49,9 \pm 3,9$ & $53,4 \pm 11,9$ & $49,2 \pm 4,1$ \\
Télévision + ordinateur en week-end et jours fériés (min/jour) & $266,3 \pm 13,3$ & $290,6 \pm 37,2$ & $261,6 \pm 14,2$ \\
Sommeil en jours ouvrables (min) & $525,1 \pm 4,7$ & $537,5 \pm 10,6$ & $522,7 \pm 5,2$ \\
Sommeil en week-end et jours fériés (min) & $575,6 \pm 8,6$ & $594,4 \pm 24,5$ & $572,0 \pm 9,1$ \\
\hline \multicolumn{1}{r}{ AP = Activités Physiques ; *significativement différent des non obèses (p<0,05). } & &
\end{tabular}


Tableau 6 : Caractéristiques socioéconomiques des ménages des sujets d'étude.

\begin{tabular}{lcccc}
\hline & & Ensemble & $\begin{array}{c}\text { Enfants } \\
\text { obèses }\end{array}$ & Enfants non-obèses \\
\hline $\mathbf{N}$ & & $\mathbf{1 0 0}$ & $\mathbf{1 6}$ & $\mathbf{8 6}$ \\
\hline $\begin{array}{l}\text { Montant moyen argent } \\
\text { (FCFA/jour)* }\end{array}$ & poche & $186,3 \pm 12,5$ & $328,1 \pm 36,2$ & $159,2 \pm 11,0$ \\
Possession de biens (\%)* & & & & \\
$\quad$ Faible & & & \\
$\quad$ Moyen & 33,0 & 6,2 & 38,1 \\
Elevé & 32,0 & 25,0 & 33,3 \\
Profession du père (\%)* & 35,0 & 68,8 & 28,6 \\
Cadre & & & \\
Employé & 37,2 & 46,7 & 35,4 \\
$\quad$ Commerçant/chef d'entreprise & 18,1 & 13,3 & 19,0 \\
Artisan & 17,0 & 33,3 & 13,9 \\
$\quad$ Profession intermédiaire & 13,8 & 0,0 & 16,5 \\
\hline
\end{tabular}

* Différence statistiquement significative entre enfants obèses et enfants non obèses $(p<0,05)$.

\section{DISCUSSION}

La présente étude a été mise en œuvre pour analyser les facteurs socioéconomiques et de mode de vie associés à l'obésité chez les écoliers de 7 à 13 ans, inscrits dans les écoles primaires privées de Cotonou.

\section{Etat nutritionnel des enfants}

Les résultats ont montré qu'il n'y a pas de différence significative entre l'âge moyen des garçons et celui des filles. Il en est de même pour les groupes d'enfants obèses et non-obèses. Toutefois, la taille, le poids, le tour de bras, le tour de taille, le tour de hanche, les plis cutanés et l'IMC des enfants obèses sont statistiquement supérieurs à ceux des enfants non obèses.

La prévalence de l'obésité a été évaluée à $16 \%$ et celle de la maigreur à $24 \%$. La prévalence de l'obésité est similaire à celle obtenue $(15,8 \%)$ dans une étude réalisée sur des enfants scolarisés de 7 à 9 ans en France en 2000 (Usen/InVS/Cnam, 2004). Elle est également comparable à celle obtenue chez les hommes et les femmes de 25 à 60 ans (18\%) dans la ville de Cotonou (Sodjinou et al, 2008). Ces résultats mettent en évidence l'effectivité de la malnutrition par surcharge dans un environnement où persiste encore la malnutrition par carence. Plusieurs auteurs ont noté la coexistence de ces deux formes de malnutrition dans les pays en transition nutritionnelle (Sodjinou et al., 2008; Ntandou, 2009 ; Delisle et al., 2012).

L'analyse des prévalences par sexe montre que l'obésité concerne plus de garçons (23\%) que de filles (10\%) sans que la différence entre sexe ne soit statistiquement significative. Ce résultat est également comparable à celui obtenu en France (Usen/InVS/Cnam, 2004).

\section{Alimentation des enfants}

Les repas consommés par les enfants sont généralement constitués des plats principaux à base de céréales ou de racines et tubercules (pâtes de maïs, pâtes alimentaires, riz, igname, manioc), accompagnés par des sauces qui sont très souvent riches en huile (friture de tomate, sauces légumes feuille à l'huile de palme, sauce de noix de palme, sauce d'arachide, etc.) et par des aliments frits (viandes, poissons, pâtés, beignets de blé, ignames frites, bananes frites).

On note une similitude dans la fréquence de consommation de certains 
groupes d'aliments (céréales, huiles et graisses, épices et condiments, légumes). En revanche, les groupes d'aliments tels que viandes et produits carnés, lait et produits laitiers, sucres et produits sucrés, sont davantage consommés par les enfants obèses que par les enfants non-obèses. L'accès à des aliments protéinés et sucrés serait fortement lié, dans les pays en développement, au pouvoir économique des individus ou des ménages (FAO, 2013).

Les résultats de l'étude mettent aussi en évidence la faible consommation des fruits dans les deux groupes d'enfants en particulier chez les enfants obèses (seulement 13\%). Ceci pose un problème de qualité de l'alimentation. Les enfants qui consomment trop peu de fruits et légumes frais s'exposent précocement aux maladies chroniques surtout lorsqu'ils vivent dans un environnement fortement influencé comme les villes où le stress et la pollution atmosphérique sont majeurs. Il est alors à craindre l'apparition précoce des maladies cardiovasculaires, de l'hypertension et du diabète au sein de la population (Sodjinou et al., 2008 ; Delisle et al., 2012). Le traitement de l'obésité et de ses complications métaboliques constituent un problème sociosanitaire et économique dont le poids est d'autant plus lourd que la maladie est précoce et que les individus vivent avec (OMS, 2003a et b).

Presque tous les enfants aiment les friandises et près de $65 \%$ prennent leur repas devant la télévision quand ils sont à la maison. $\mathrm{Ce}$ comportement semble influencer positivement la survenue de l'obésité chez les élèves au Canada (Liang et al., 2009), mais les résultats de cette étude ne montrent pas de lien avec la présence ou non de l'obésité.

La différence significative observée entre la fréquence moyenne des repas des enfants obèses $(5,4 \pm 0,3)$ et celle des enfants non-obèses $(4,7 \pm 0,1)$ montre que les enfants obèses mangent un peu plus fréquemment que les enfants non-obèses.

Tous les apports de nutriments des enfants obèses sont statistiquement plus élevés que pour les enfants non-obèses. Ceci s'explique par le fait que les enfants obèses consomment plus de quantité d'aliments que les enfants non-obèses, l'énergie moyenne ingérée par les enfants obèses $(14,1 \pm 9,1 \mathrm{MJ})$ étant le double de celle ingérée par les enfants non obèses $(6,5 \pm 5,5 \mathrm{MJ})$. L'apport énergétique moyen journalier de l'ensemble des sujets (7,8 MJ) est 1,5 fois plus élevé que celui estimé au sein des enfants de 6 à 8 ans (5,0 MJ) en milieu rural au nord du Bénin (Mitchikpè et al., 2007). La différence entre les deux études serait due à l'âge plus jeunes des enfants du nord mais aussi au milieu d'étude. En effet, en milieu rural, les populations ont moins facilement accès à une alimentation variée qu'en milieu urbain, et en ce qui concerne ce dernier, l'alimentation est souvent dominée par des produits très gras et sucrés. Ceci peut être constaté à travers le régime alimentaire déséquilibré des enfants, avec un déséquilibre plus prononcé chez les enfants obèses. En effet, les lipides contribuent pour $51 \%$ de l'apport énergétique total chez les enfants obèses contre $38 \%$ chez les enfants non-obèses $(p=0,05)$. Ces apports dépassent largement les recommandations qui situent entre 20 et $30 \%$ la contribution de l'apport énergétique total pour une alimentation équilibrée (OMS, 2003a). La contribution des glucides à l'apport énergétique total est également largement insuffisante chez les enfants obèses (39\% contre 55 à $75 \%$ selon les recommandations).

Les enfants obèses consomment plus de graisses polyinsaturées et de graisses saturées que les enfants non-obèses avec une différence statistiquement significative. La contribution des graisses polyinsaturées à l'apport énergétique total est au-delà des recommandations (6-10\%) (FAO/OMS, 2008) dans les deux groupes d'enfants. Il en est de même pour la contribution des graisses saturées à l'apport énergétique total (>10\%) chez les enfants obèses. De plus, ces derniers présentent un apport moyen en cholestérol dépassant les normes et très supérieur à celui des enfants non-obèses. Compte tenu des liens 
établis entre la forte consommation de graisses saturées et de cholestérol et les problèmes de santé, les enfants obèses sont exposés au risque de souffrir à un âge précoce des maladies cardiovasculaires, d'hypertension artérielle et de dyslipidémie.

Le score moyen de diversité alimentaire est de 8,25 chez les enfants obèses et de 7,61 chez les enfants non-obèses. L'apport alimentaire est donc plus varié chez les enfants obèses, la proportion d'enfants obèses ayant un niveau de diversité alimentaire très élevé (44\%) étant le double de celle des enfants non-obèses. L'accès des enfants obèses à une alimentation plus diversifiée pourrait s'expliquer par les caractéristiques socioéconomiques de de leurs ménages.

\section{Activités physiques et sédentarité des enfants}

L'activité physique et la sédentarité sont deux éléments mis en relation avec la prévalence du surpoids et de l'obésité vu qu'elles influencent la dépense énergétique totale journalière des enfants. Très peu d'enfants obèses vont à l'école à pieds $(6 \%)$ alors que plus de la moitié des enfants nonobèses utilisent la marche comme moyen pour se rendre à l'école (54\%). De plus, la durée moyenne du trajet maison-école parcourue quotidiennement à pied par les enfants nonobèse $(47,4 \mathrm{~min} / \mathrm{j})$ est plus du double de celle des enfants obèses ( $20 \mathrm{~min} /$ jour). Ceci montre une réduction de la pratique d'activité physique au sein des enfants obèses qui pourrait s'expliquer par le changement des habitudes dû aux modifications de l'environnement et à l'amélioration du statut socioéconomique des ménages (Owen et al., 2000; Maffeis et Castellani, 2007). Makoutodé et al. (2017) ont aussi trouvé que la réduction de l'activité physique est un facteur associé à la surcharge pondérale chez les adolescents de 10 à 19 ans dans la ville de Ouidah au Bénin.

Certains parents inscrivent leurs enfants dans des activités sportives structurées en dehors de l'école parce que probablement conscients des effets néfastes de l'obésité ou le font par conformisme. Cette pratique de l'activité physique en dehors du collège est à encourager et les parents doivent veiller à ce que les enfants participent effectivement aux séances. Il n'existe pas une recommandation précise sur la durée de l'activité physique permettant de prévenir une prise de poids préjudiciable à la santé, mais environ $30 \mathrm{~min}$ d'activité physique modérée par jour vise la réduction des maladies cardio-vasculaires et la mortalité en général (INSERM, 2008 ; OMS, 2003 a). Pour un meilleur effet de la pratique de l'activité physique, les durées pourraient être scindées en plusieurs périodes comme le suggèrent Saris et al. (2003).

En général, en jours ouvrables comme en week-end et jours fériés, les enfants obèses consacrent quotidiennement plus de temps aux activités sédentaires que les enfants nonobèses, même si aucune différence significative n'a été trouvée entre les deux groupes d'enfants. L'ordinateur et les jeux vidéo occupent deux fois plus de temps chez les enfants obèses que chez les enfants nonobèses. Ces constats soulèvent les risques de développement d'une vie solitaire chez les enfants obèses à cause de l'augmentation de leur isolement et de l'absence d'interaction avec les enfants du voisinage. Ceci pourrait avoir des conséquences psychologiques chez ces enfants.

Aucune association n'a été observée entre la durée moyenne du sommeil (9 heures par jour en semaine et un peu moins de 10 heures par jour en week-end et jours fériés) et l'obésité chez les enfants. Ces résultats sont différents de ceux rapportés par Marshall et al. (2008) qui ont trouvé une relation linéaire négative entre la durée du sommeil et l'obésité chez l'enfant. Ils sont également différents de ceux rapportés par Makoutode et al. (2017) qui ont trouvé que le sommeil est un facteur significativement associé à la surcharge pondérale chez les adolescents de 10 à 19 ans dans la ville de Ouidah au Bénin. Les résultats de notre étude peuvent s'expliquer par le fait 
que probablement l'heure de coucher est encore sous le contrôle parental si bien que les enfants dorment et se réveillent généralement à des heures régulières.

\section{Niveau socioéconomique des ménages}

Les résultats montrent que plus de deux tiers des enfants obèses vivent dans des ménages ayant un score élevé de possession de biens, alors que les enfants non-obèses sont dans des ménages répartis de façon quasi uniforme dans les trois catégories de possession de biens. L'obésité est associée à la possession dans le ménage d'un ordinateur, de jeux-vidéos, de groupe électrogène, d'une dalle comme type de plafond. Ces résultats confirment l'influence du niveau économique du ménage sur la prévalence de l'obésité au sein des enfants. En fait, dans les pays en développement tels que le Bénin, les plus riches ont un niveau de vie élevé avec une plus grande possibilité d'accès aux produits alimentaires de tout genre, ce qui entraîne aussi une modification des habitudes alimentaires (OMS, 2003a). De plus, une relation entre la profession du père et la présence de l'obésité a été mise en évidence $(p<0,05)$. Environ $80 \%$ des enfants obèses ont des pères cadres, commerçants ou chefs d'entreprise. Aucun enfant d'artisans n'est obèse. La relation entre l'obésité et le niveau socioéconomique se traduit également par la différence entre les montants de l'argent de poche donnés aux enfants. L'argent reçu par les enfants obèses $(328,1 \pm 36,2$ FCFA) est en moyenne deux fois plus élevé que celui reçu par les enfants non-obèses $(159,2 \pm 11,0$ FCFA). Cet argent est généralement utilisé pour acheter des snacks tels que les biscuits (sucrés, salés, chocolatés ou à la crème), les chips, le yaourt, le jus de bissap, les crèmes glacées, le chocolat, les produits de pâtisseries, les aliments frits (igname, banane, beignets d'haricot, beignets de blé ou de maïs). La consommation fréquente de ces produits expose davantage les enfants aux risques d'obésité.

\section{Conclusion}

La faible consommation des fruits, mais la consommation élevée d'énergie, des lipides, des graisses saturées et du cholestérol, le déséquilibre entre lipides, protéines et glucides, la diminution des activités physiques intenses en faveur des activités sédentaires constituent, en lien avec le statut socioéconomique du ménage, les facteurs de risques de l'obésité et des maladies nontransmissibles chez les enfants d'âge scolaire dans la ville de Cotonou. Des séances de sensibilisation et de formation sur une bonne alimentation ainsi que les conséquences de la malnutrition, organisées à l'endroit des élèves, des parents ainsi que des responsables d'écoles pourrait aider à prévenir l'obésité chez les enfants d'âge scolaire. Il serait également nécessaire de mettre en place un système de surveillance de la malnutrition pour les enfants d'âge scolaire.

\section{CONFLIT D'INTERETS}

Les auteurs déclarent qu'il n'y a aucun conflit d'intérêts entre eux.

\section{CONTRIBUTIONS DES AUTEURS}

JAF a participé à la conception de l'étude, sa mise en œuvre, l'analyse statistique des données, l'interprétation des résultats et à la rédaction de la première mouture de l'article ; CESM a participé en tant qu'auteur correspondant à la conception de l'étude, à l'analyse statistique des données, à l'interprétation des résultats ainsi qu'à la rédaction de l'article; DJH a participé à la conception de l'étude et à la relecture du manuscrit.

\section{REFERENCES}

Afrifa-Anane E, Agyemang C, Codjoe SNA, Ogedegbe G and Aikins AG. 2015. The association of physical activity, body mass index and the blood pressure levels among urban poor youth in Accra, Ghana. BMC Public Health, 15: 269. DOI: https://doi.org/10.1186/s12889015-1546-3. 
Cole TJ, Bellizi MC, Flega KM, Dietz WH. 2000. Establishing a standard definition for child overweight and obesity worldwide: international survey. British Medical Journal, 320:1-6.

Cole TJ, Flegal KM, Nicholls D, Jackson AA. 2007. Body Mass Index cut offs to define thinness in children and adolescents international survey. British Medical Journal, 335: 194-197. DOI: https://doi.org/10.1136/bmj.39238.39944 4.55 .

Delisle H, Ntandou-Bouzitou G, Agueh V, Sodjinou R, Fayomi B. 2012. Urbanisation, nutrition transition and cardiometabolic risk: the Benin study. British Journal of Nutrition, 107(10): 1534-1544.

DOI: 10.1017/S0007114511004661

Ministère de la Santé du Bénin. 2008. Rapport final de l'enquête STEPS au Bénin. Juin 2008. Ministère de la Santé : Cotonou, p.126.

Ministère de la Santé du Bénin. 2016. Rapport final de l'enquête pour la surveillance des facteurs de risque des maladies non transmissibles par l'approche "STEPSwise" de l'OMS. ENQUETE 'STEPS 2015' au Bénin. Ministère de la Santé : Cotonou, p.152.

FAO. 2011. Profil Nutritionnel du Bénin. Division de la Nutrition et de la Protection des Consommateurs. FAO: Rome ; 62.

FAO. 2013. Guidelines for Measuring Household and Individual Dietary Diversity. FAO: Rome, Italy.

FAO/OMS. 2008. Interim Summary of Conclusions and Dietary Recommendations on Total Fat \& Fatty Acids. Joint FAO/WHO Expert Consultation on Fats and Fatty Acids in Human Nutrition, November 10-14, 2008, WHO HQ, Geneva. 2010; http://www.fao.org/ag/agn/nutrition/docs /Fats and Fatty Acids Summary.pdf, P.15.
Gibson RS, Ferguson EL. 2008. An Interactive 24-hour Recall for Assessing the Adequacy of Iron and Zinc Intakes in Developing Countries. Harvest Plus Technical Monograph 8. International Life Sciences Institute : Washington DC.

Gouvernement du Bénin. 2009. Analyse globale de la vulnérabilité, de la sécurité alimentaire et de la nutrition AGVSAN. Bénin, p.152.

Houweling TAJ, Kunst AE, Mackenbach JP. 2003. Measuring health inequality among children in developing countries: does the choice of the indicator of economic status matter? International Journal Equity Health, 2(1): 8. DOI: 10.1186/1475-9276-2-8.

INSERM. 2008. Activité physique : contextes et effets sur la santé. Rapport. Paris : Les éditions Inserm, 2008, XII - 811 p. (Expertise collective). http://hdl.handle.net/10608/97".

Liang T, Kuhle S, Veugelers PJ. 2009. Nutrition and body weights of Canadian children watching television and eating while watching television. Public Health Nutrition, 12(12): 2457-2463. DOI: $10.1017 / \mathrm{S} 1368980009005564$.

Maffeis C, Castellani M. 2007. Physical activity: An effective way to control weight in children? Nutrition, Metabolism \& Cardiovascular Diseases; 17(5): $\quad 394-408 . \quad$ DOI: https://doi.org/10.1016/j.numecd.2006.0 8.006

Makoutodé A, Saïzonou J, Glele Ahanhanzo Y, Sossa CJ, Agueh V. 2017. Prévalence et facteurs associés à la surcharge pondérale chez les adolescents dans une ville secondaire au Bénin. Int. J. Biol. Chem. Sci., 11(2): 798-805. DOI : https://dx.doi.org/10.4314/ijbcs.v11i2.21

Marshall NS, Glozier N, Grunstein RR. 2008. Is sleep duration related to obesity? A critical review of the epidemiological evidence. Sleep Medicine Reviews, 
12(4): 289-298. DOI: 10.1016/j.smrv.2008.03.001.

Mitchikpe CES, Dossa RAM, Ategbo EAD, van Raaij JMA, Kok FJ. 2007. Seasonal variation in food pattern but not in energy and nutrient intakes of rural Beninese school-age children. Public Health Nutrition, 12: 414-422. DOI: 10.1017/S1368980008002929.

Ntandou G. 2009. Transition nutritionnelle et facteurs de risque de maladies cardiovasculaires au Bénin. Etude dans la ville secondaire de Ouidah et sa périphérie rurale. Thèse de Doctorat, Université de Montréal, Canada, p.401.

OMS. 2003a. Régime alimentaire, nutrition et prévention des maladies chroniques. Rapport de consultation OMS/FAO, Série de Rapports techniques. $\mathrm{N}^{\circ} 916$. Genève : Organisation mondiale de la santé. p. 142.

OMS. 2003b. Obésité : prévention et prise en charge de l'épidémie mondiale. Rapport de consultation OMS, Série de Rapports techniques. $\quad \mathrm{N}^{\circ}$ 894. Genève : Organisation mondiale de la santé. p.296.

OMS. 2010. Stratégies de prévention de l'obésité de l'enfant dans la population : rapport du forum et de la réunion technique de l'OMS, Genève, 15-17 décembre 2009. Organisation mondiale de la santé, Suisse.

OMS. 2014. Global status report on Noncommunicable diseases. Organisation mondiale de la santé, Suisse.

Owen N, Leslie E, Salmon J, Fotheringham M. 2000. Environmental determinants of physical activity and sedentary behavior. Exerc. Sport Sci. Review, 28(4):153-8.

Saris WHM, Blair SN, van Baak MA, Eaton SB, Davies PSW, Di Pietro L, Fogelholm M, Rissanen A, Schoeller D, Swinburn B, Tremblay A, Westerterp KR, Wyatt H. 2003. How much physical activity is enough to prevent unhealthy weight gain? Outcome of the IASO 1st Stock Conference and consensus statement. Obesity Reviews, 4: 101-114. DOI: $\quad 10.1046 / \mathrm{j} .1467-$ 789X.2003.00101.x.

Sodjinou R, Agueh V, Fayomi B, Delisle H. 2008. Obesity and cardio-metabolic risk factors in urban adults of Benin: Relationship with socio-economic status, urbanisation and lifestyle patterns. $B M C$ Public Health, 8(1): 84. DOI: 10.1186/1471-2458-8-84.

Usen/InVS/Cnam. 2004. Surpoids et obésité chez les enfants de 7 à 9 ans. Paris, France. P.40. 